\title{
Impacto do Tratamento com GH sobre as Funções Tireoidiana e Adrenal
}

\begin{abstract}
RESUMO
O estado somatotrófico é modulador importante dos eixos tirotrófico e corticotrófico. Enquanto a reposição somatotrófica em pacientes com deficiência de GH aumenta a conversão do hormônio inativo $\left(T_{4}\right)$ na sua forma ativa $\left(T_{3}\right)$, aumentando dessa forma a ação biológica do hormônio tireoidiano, a mesma reposição induz no eixo corticotrófico a conversão de cortisol, hormonalmente ativo, em cortisona, que é biologicamente inativa. Nessa revisão, foram discutidos os efeitos do GH nesses dois eixos hormonais, os possíveis mecanismos e as implicações clínicas no manejo dos pacientes com hipopituitarismo. (Arq Bras Endocrinol Metab 2008; 52/5:889-900)
\end{abstract}

Descritores: Hormônio de crescimento; Levotiroxina, Glicocorticóides, Hipopituitarismo; Desiodases

\section{ABSTRACT}

hGH Treatment Impact on Adrenal and Thyroid Functions.

Somatotrophic status is a major determinant of both thyrotrophic and corticotrophic axis. In growth hormone deficient patients, somatotrophic replacement increases the conversion rate of the inactive form of the thyroid hormone $\left(T_{4}\right)$ to its active form $\left(T_{3}\right)$, whereas the same replacement induces the conversion of cortisol, which is hormonally active, in cortisone, its inactive form. This review details the effects of $\mathrm{GH}$ on these two hormonal axis, possible mechanisms and clinical implications for the management of hypopituitary patients. (Arq Bras Endocrinol Metab 2008; 52/5:889-900)

Keywords: Growth hormone; Levotoxin; Glicocorticotrophic; Hypopituitarism; Desiodases

\section{INTRODUÇÃO}

$\mathrm{O}$ HIPOTIREOIDISMO CENTRAL É UMA deficiência freqüente entre pacientes com doença hipotálamo-hipofisária. Seu tratamento consiste da terapia de reposição hormonal com levotiroxina. Entretanto, diferentemente do hipotireoidismo primário, em que o TSH sérico é um marcador acurado de eutireoidismo tecidual, os médicos tem de se fundamentar em medidas séricas dos hormônios tireoidianos (HT), $\mathrm{T}_{4}$ e $\mathrm{T}_{3}$. Na falta de um marcador útil de ação de HT, o tratamento do hipotireoidismo central tem como base recomendações tradicionais e empíricas de manter os níveis séricos de $\mathrm{T}_{4}$ livre na metade superior do intervalo de referência, a fim de evitar o hipotireoidismo na maioria dos pacientes. Esse objetivo geralmente é estabelecido independentemente do estado somatotrófico. Entretanto, a deficiência de hormônio de crescimento (GH), que é comum nesses pacientes, sabidamente induz a mudanças profundas no meta- revisão

\author{
Manoel R. A. Martins \\ JULIO ABUCHAM
}

Serviço de Endocrinologia e Diabetes da Universidade Federal do Ceará, Fortaleza, CE, Brasil (MRAM); Disciplina de Endocrinologia do Departamento de Medicina da Escola Paulista de Medicina da Universidade Federal de São Paulo, SP, Brasil (JA).

Recebido em 26/5/2008

Aceito em 30/5/2008 
bolismo de HT, as quais devem ser levadas em consideração durante o manejo da reposição com levotiroxina. Além disso, o objetivo da reposição com levotiroxina se baseia na medida do $\mathrm{T}_{4}$ sérico, que é um prohormônio, ao passo que a forma bioativa do HT é o $\mathrm{T}_{3}$.

Esta revisão discute o impacto da reposição com GH no metabolismo do HT, os mecanismos possíveis desses efeitos e a implicação clínica no manejo do hipotireoidismo central.

\section{HIPOTIREOIDISMO CENTRAL (HC)}

O HC é uma desordem comum em pacientes com doença hipotálamo-hipofisária. Ele é caracterizado por níveis séricos baixos de HT por causa da estimulação diminuída de uma glândula tiróide normal por um TSH diminuído ou biologicamente menos ativo $(1,2)$. O HC inclui o hipotireoidismo hipotalâmico (terciário), causado por deficiência de TRH, o hipotireoidismo hipofisário (secundário), em razão da diminuição de $\mathrm{TSH}$, assim como o hipotireoidismo determinado por ambos os componentes. Os mecanismos envolvidos na patogênese do HC incluem diminuição dos tirotrofos funcionantes, redução da síntese/secreção de TRH hipotalâmico e redução da potência biológica do TSH (2-4).

As causas mais comuns de $\mathrm{HC}$ adquirido incluem lesões tumorais no hipotálamo e/ou hipófise e o tratamento dessas lesões, tanto cirúrgico quanto radioterápico. Mais recentemente, tem sido demonstrada a importância do dano cerebral por causa de trauma crânincefálico (5), hemorragia subaracnóidea de aneurisma (5), acidente vascular isquêmico (6), neurocirurgia (7) e irradiação craniana (8) como causa freqüente e anteriormente subestimada de disfunção hipotálamohipofisária. Outras causas incluem doenças granulomatosas e necrose hipofisária pós-parto (síndrome de Sheehan). Nas formas hereditárias, o HC está freqüentemente associado à neurohipófise ectópica. Mutações levando a causas raras de HC familiar foram descritas nos genes que codificam a subunidade $\beta$ do TSH (9-13), o receptor de TRH (14) e o fator de transcrição hipofisário (Pitl) ou seu profeta (POUlF1) $(15,16)$.

O quadro clínico é semelhante ao de pacientes com hipotireoidismo primário, entretanto geralmente é mais leve e o bócio está ausente. Podem ser observados fadiga fácil, sonolência, ganho ponderal leve, intolerância ao frio, constipação, pele seca e bradicardia. Em crianças, o achado mais notável do HC é a diminuição da velocidade de crescimento. Deve ser notado que muitos sinais e sintomas do HC não são específicos, podem ser leves e podem ser confundidos com os de outras deficiências hipofisárias. Sendo assim, o HC tem de ser avaliado laboratorialmente na maioria dos pacientes com doença hipotálamo-hipofisária.

Ao diagnóstico, os níveis séricos de TSH podem estar diminuídos, normais ou discretamente aumentados [geralmente menores que $10 \mathrm{mUI} / \mathrm{mL}(2)]$ na presença de níveis séricos baixos de $\mathrm{T}_{4}$ livre. $\mathrm{O}$ teste de estímulo de TSH com TRH geralmente não é útil para o diagnóstico (17). Durante a reposição com levotiroxina, o TSH sérico consistentemente cai para níveis baixos ou indetectáveis durante a reposição fisiológica de levotiroxina (18). Dessa forma, tanto o diagnóstico quanto a adequação da reposição com levotiroxina no $\mathrm{HC}$ tem se fundamentado nos níveis séricos de $\mathrm{T}_{4}$, os quais mostram variações intra-individuais muito menores que o intervalo de referência (19).

A reposição hormonal padrão no $\mathrm{HC}$ consiste de levotiroxina por via oral. A dose de levotiroxina em adultos jovens varia entre 0,7 e $1,5 \mu \mathrm{g} / \mathrm{kg} / \mathrm{d}$ (20), com doses menores sendo geralmente usadas em pacientes mais velhos. Em crianças, doses típicas variam entre 8 e $10 \mu \mathrm{g} /$ $\mathrm{kg} / \mathrm{d}$ até os 6 meses de idade, 6 e $8 \mu \mathrm{g} / \mathrm{kg} / \mathrm{d}$ entre os 6 e 12 meses, 5 e $6 \mu \mathrm{g} / \mathrm{kg} / \mathrm{d}$ em crianças entre 1 e 5 anos, e 4 e $5 \mu \mathrm{g} / \mathrm{kg} / \mathrm{d}$ em crianças entre 6 e 12 anos (2l).

$\mathrm{O}$ alvo tradicional da reposição com levotiroxina é um $\mathrm{T}_{4}$ livre sérico na metade superior da normalidade (22), a fim de evitar o hipotireoidismo. Entretanto, como será apresentada a seguir, essa recomendação não leva em consideração os efeitos da reposição de GH no metabolismo do HT.

A deficiência de GH é a deficiência hipofisária mais comum. Assim, a maioria dos pacientes com HC também é deficiente de GH. A maioria dos pacientes com deficiência de GH é reposta com GH durante a infância. Entretanto, a maioria desses pacientes terá sua reposição de GH suspensa após atingir a altura final, e alguns outros não serão repostos por causa das contraindicações e de outros motivos.

\section{EFEITO DO GH NO METABOLISMO DO HT}

O primeiro relato do impacto clínico da reposição de GH no estado tirotrófico em crianças foi realizado há mais de 30 anos (23). Seis crianças com deficiência de GH tornaram-se hipotireóideas clínica e laboratorialmente após o início da reposição somatotrófica. Entretanto, essas crianças já apresentavam níveis séricos basais de $\mathrm{T}_{4}$ próximos do limite inferior do intervalo de refe- 
rência, o que sugere que elas já estavam em hipotireoidismo antes do início da reposição de GH. Os relatos iniciais também sugeriam que a reposição de $\mathrm{GH}$ reduzia a captação tireoidiana de iodo radioativo (24) e diminuia a resposta do TSH sérico ao TRH exógeno (25), levando à hipótese de que o $\mathrm{GH}$ poderia inibir a síntese e/ou a liberação de TSH pela hipófise $(24,25)$. Os resultados desses trabalhos iniciais sobre o efeito do GH no metabolismo periférico do HT foram muito variáveis em virtude de vários fatores, como protocolos de estudo diferentes, diversidades nos métodos analíticos usados para medir os HT e contaminação das preparações de GH com TSH, além do pequeno número de pacientes estudados. De mais a mais, a noção de que o $\mathrm{GH}$ poderia induzir hipotireoidismo era difícil de conciliar com os sabidos efeitos do $\mathrm{GH}$ em promover o crescimento e suas ações calorigênicas $(26,27)$.
Vários estudos seguintes apontaram que a administração de GH aumenta a conversão periférica de $\mathrm{T}_{4} \mathrm{em}$ $\mathrm{T}_{3}$, tanto em indivíduos normais quanto em pacientes deficientes de GH (27-35) (Tabelas l e 2). Notavelmente, esse efeito também foi demonstrado em pacientes com HC durante reposição com doses fixas de levotiroxina, tanto em crianças (33) quanto em adultos (35), descartando o mecanismo central via TRH-TSH. Estudos clínicos mostrando nenhuma mudança ou mudança transitória nos níveis de HT após reposição de GH geralmente incluíram indivíduos normais (40) ou pacientes com deficiência de $\mathrm{GH}$ isolada (37-43).

Em um estudo clínico realizado pelo nosso grupo (33), com 20 crianças com DGH avaliadas em duas situações, com e sem reposição de $\mathrm{GH}$, a reposição de $\mathrm{GH}$ induziu à queda dos níveis de $\mathrm{T}_{4}$ livre sérico e de $\mathrm{T}_{3}$ reverso e o aumento dos níveis de $\mathrm{T}_{3}$ total (Figura 1).

Tabela 1. Efeito do tratamento com GH nos níveis séricos de HT em crianças.

\begin{tabular}{|c|c|c|c|c|c|c|c|}
\hline & Ano & Pacientes & TSH & $\mathrm{T}_{4}$ & $\mathrm{~T}_{3}$ & $T_{3} r$ & $\mathrm{n}$ \\
\hline Porter (36) & 1973 & DGH & - & $=$ & - & - & 5 \\
\hline Root (25) & 1973 & DGH & $=$ & $=$ & $\uparrow$ & - & 10 \\
\hline Lippe (23) & 1975 & DGH & - & $\downarrow$ & $\downarrow$ & - & 6 \\
\hline Rubio (37) & 1976 & DGH & $=$ & $=$ & $=$ & - & 12 \\
\hline Sato (28) & 1977 & DGH & - & $\downarrow$ & $\uparrow$ & - & 8 \\
\hline Cacciari (38) & 1979 & DGH & $=$ & $=$ & $=$ & $=$ & 24 \\
\hline Rezvani (29) & 1981 & DGH & $=$ & $=$ & $\uparrow$ & $\downarrow$ & 7 \\
\hline Pirazzoli (34) & 1992 & DGH & $\downarrow$ & $\downarrow$ & $\uparrow$ & - & 57 \\
\hline Municchi (39) & 1992 & DGH & $=$ & $=$ & $=$ & - & 23 \\
\hline Rose (40) & 1995 & Normal & $=$ & $=$ & $=$ & - & 9 \\
\hline Amato* (41) & 1996 & DGH & $=$ & $=$ & $=$ & $=$ & 9 \\
\hline Hussain (42) & 1996 & $\mathrm{NI} / \mathrm{DGH}$ & - & $=$ & $\uparrow$ & - & 8 \\
\hline Wyatt** (43) & 1998 & DGH & - & $\downarrow$ & $\uparrow$ & $\downarrow$ & 15 \\
\hline Portes (33) & 2000 & DGH & $=$ & $\downarrow$ & $\uparrow$ & $\downarrow$ & 20 \\
\hline Giavoli (44) & 2003 & DGH & $=$ & $\downarrow$ & $\uparrow * * *$ & - & 26 \\
\hline Kalina-Faska** (45) & 2004 & DGH & $\downarrow$ & $\downarrow$ & $\uparrow$ & - & 32 \\
\hline Seminara** (46) & 2005 & DGH & $=$ & $\downarrow$ & $\uparrow$ & - & 19 \\
\hline Martins (32) & 2007 & $\mathrm{DGH}$ & $=$ & $\downarrow$ & $\uparrow$ & - & 32 \\
\hline
\end{tabular}


Tabela 2. Efeito do tratamento com GH nos níveis séricos de HT em adultos.

\begin{tabular}{|c|c|c|c|c|c|c|c|}
\hline & Ano & Pacientes & TSH & $\mathrm{T}_{4}$ & $\mathrm{~T}_{3}$ & $T_{3} r$ & $\mathrm{n}$ \\
\hline Grunfeld (30) & 1988 & Homens normais & $\downarrow$ & $\downarrow$ & $\uparrow$ & $=$ & 20 \\
\hline Jorgensen (35) & 1989 & $\mathrm{DGH}$ & $=$ & $\downarrow$ & $\uparrow$ & - & 22 \\
\hline Binnerts (47) & 1992 & DGH & - & $\downarrow$ & $\uparrow$ & $\downarrow$ & 8 \\
\hline Moller (27) & 1992 & Homens normais & $=$ & $=$ & $\uparrow$ & $\downarrow$ & 14 \\
\hline Bengtsson (48) & 1993 & DGH & - & $\downarrow^{*}$ & $\uparrow$ & - & 10 \\
\hline Jorgensen (31) & 1994 & DGH & $\downarrow$ & $\downarrow^{* *}$ & $\uparrow$ & $\downarrow$ & 8 \\
\hline Jorgensen (49) & 1994 & Mulheres obesas & $=$ & $\downarrow$ & $\uparrow$ & $\downarrow$ & 10 \\
\hline Porretti (50) & 2002 & DGH & $=$ & $\downarrow$ & $=$ & $\downarrow$ & 66 \\
\hline Giavoli (51) & 2003 & DGH & $=$ & $\downarrow$ & $=$ & - & 20 \\
\hline Agha (8) & 2007 & DGH & $=$ & $\downarrow$ & $\uparrow * * *$ & - & 243 \\
\hline
\end{tabular}

- = não medido; ${ }^{*}=$ mudanças transitórias, revertidas ao final do estudo; ${ }^{* *}=p=0,06$; ${ }^{* * *}=$ tendência.

(a)

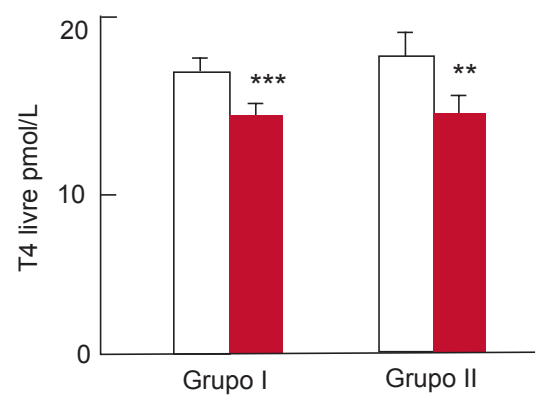

(b)

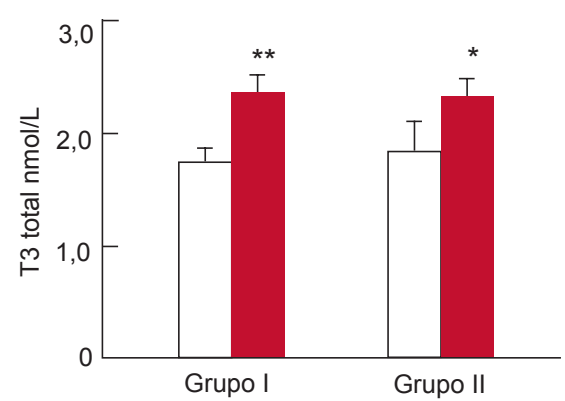

(c)

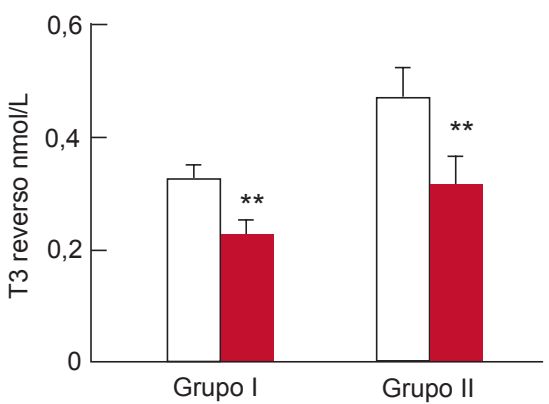

${ }^{*} p<0,05 ;{ }^{* *} p<0,01 ;{ }^{* *} p<0,001$. (Portes e cols., 2000 [33]).

Figura 1. Niveis séricos de (a) T4 livre, (b) T3 total e (c) T3 reverso durante a reposição de GH (colunas brancas) e sem GH (colunas pretas) em crianças com DGH agrupadas de acordo com o tratamento com levotiroxina e níveis em grupo I (sem reposição de levotiroxina e TSH > 0,4 mU/l, $n=12$ ) e grupo $\|$ (em reposição de levotiroxina e TSH < 0,05 $\mathrm{mU} / \mathrm{l}, \mathrm{n}=8$ ). Os resultados são apresentados em média \pm EP.

\section{EFEITO DO GH NO METABOLISMO PERIFÉRICO DOS HT: MECANISIMOS POSSÍVEIS}

\section{Metabolismo periférico dos HT}

Em estudos iniciais, o efeito central do GH na síntese e na liberação de TSH foi aventado $(24,25)$. Entretanto, foi demonstrado, posteriormente, que as mudanças nos níveis de HT também estavam presentes em pacientes durante a reposição de doses fixas de levotiroxina, sugerindo que o mecanismo envolvido não abrange o eixo hipotálamo-hipófise $(33,35)$. Além disso, a queda sincrônica nos níveis de $\mathrm{T}_{4}$ e $\mathrm{T}_{3}$ reverso acoplada com o aumento dos níveis de $\mathrm{T}_{3}$ sérico sugere que a reposição de $\mathrm{GH}$ induz mudanças no metabolismo periférico do HT (33).
$\mathrm{O} \mathrm{T}_{4}$ é um prohormônio que precisa ser ativado via desiodação na sua forma bioativa, $\mathrm{o} \mathrm{T}_{3}$, para iniciar sua ação. As vias de ativação e desativação dos $\mathrm{HT}$ estão sob o controle de três desiodases, tipos I a III (52). Essas três enzimas são selenoproteínas que estão presentes em todos os vertebrados e podem ativar ou inativar os HT, dependendo se agem no anel fenólico ou tirosil das iodotironinas (53). A desiodação do $\mathrm{T}_{4} \mathrm{em}$ $\mathrm{T}_{3}$ ocorre no anel fenólico (exterior) da molécula de $\mathrm{T}_{4}$ e é catalisada pela desiodase tipo 1 ou pela tipo 2. Por outro lado, essa ativação do $\mathrm{T}_{4}$ pode ser evitada e o $\mathrm{T}_{3}$ pode ser inativado de maneira irreversível pela desiodação do anel tirosil (interior), reação que é catalisada pelas desiodases tipo 3 e também pela tipo 1. A D2 gera $\mathrm{T}_{3}$ via desiodação do $\mathrm{T}_{4}$. Em contraste, a $\mathrm{D} 3$ inativa o $\mathrm{T}_{3}$ e de maneira menos importante evita que o $\mathrm{T}_{4}$ seja 
ativado. Finalmente, a Dl ativa ou inativa o $\mathrm{T}_{4}$ de maneira equimolar, e seu papel em condições fisiológicas ainda não foi elucidado (54).

A Dl foi identificada no fígado e nos rins de ratos e humanos, e geralmente é assumido que essa enzima seja a principal fonte de $\mathrm{T}_{3}$ plasmático em humanos. A descoberta do RNAm de D2 e sua atividade no musculoesquelético humano sugerem que a D2 também possa ser fonte significativa da produção de $\mathrm{T}_{3}$ plasmático em humanos, e foi sugerido que a D2 seja fonte importante de $\mathrm{T}_{3}$ plasmático em humanos em eutireoidismo (55), ao passo que a Dl pode ser a fonte mais importante durante o hipertireoidismo (20,56-65).

Os efeitos do GH no metabolismo do HT são consistentes com a ativação aumentada da Dl e/ou da D2, e/ou ativação diminuída da D3. Esses efeitos estimulatórios do GH no metabolismo do HT foram demonstrados em várias espécies de vertebrados $(20,64)$. Em galinhas, foram associados à diminuição da atividade da $\mathrm{D} 3(53,54)$. Entretanto, esses modelos experimentais não são bons preditores do mecanismo em humanos. Além disso, o efeito do $\mathrm{GH}$ ao diminuir a atividade de $\mathrm{D} 3$ não explicaria a diminuição nos níveis de $\mathrm{T}_{4}$, conforme observado em estudos em humanos $(28,30-34,44,47,49,50)$.

\section{Efeito do estado somatotrófico no metabolismo dos HT}

O GH induz a síntese hepática e a secreção do fator de crescimento insulina-símile (IGF-1) (66). Apesar de muitos efeitos do GH serem mediados por IGF-1, o GH também exerce alguns efeitos diretos e eventualmente opostos ao do IGF-1, em especial no metabolismo dos carboidratos. Os receptores de GH estão presentes virtualmente em todos os tecidos. Em humanos, níveis elevados de expressão de RNAm do receptor de $\mathrm{GH}$ foram demonstrados no fígado, no tecido adiposo, nos músculos e nos rins (67).

Embora a conversão dos HT ocorra na maioria dos tecidos, os principais sítios de conversão são o fígado, os rins e o musculoesquelético. O fígado é considerado o sítio mais importante de conversão de $\mathrm{T}_{4} \mathrm{em} \mathrm{T}_{3}$. Os hepatócitos, ao contrário do que ocorre nos rins e nas células musculares esqueléticas, não expressam receptores funcionais para IGF-1 (68).

Se é o GH ou o IGF-1 principal efetor das mudanças que o estado somatotrófico exerce no metabolismo dos HT foi avaliado. Em pacientes com DGH, apesar de o tratamento com IGF-1 ser capaz de induzir o au- mento dos níveis de $\mathrm{T}_{3}$ séricos, efeito mais pronunciado é observado após a reposição de GH (42), sugerindo que este induz a tais mudanças além do mecanismo via IGF-1. Em um outro estudo nenhum efeito nos níveis séricos de $\mathrm{T}_{3}$ foi observado após a administração de IGF-1 em pacientes com insensibilidade ao $\mathrm{GH}$ em razão de mutações no receptor de GH (69). Em conjunto, esses resultados sugerem que os efeitos da reposição de GH nos níveis circulantes de HT parecem ser mediados predominantemente pelo efeito direto do $\mathrm{GH}$.

\section{CONDUTA CLÍNICA TRADICIONAL}

A maior parte das recomendações sobre a reposição de levotiroxina em pacientes com HC sugere que os níveis de $T_{4}$ séricos devem ser mantidos na metade superior da faixa de referência (22). O estado somatotrófico geralmente não é levado em consideração, de tal forma que na prática clínica essas recomendações têm sido aplicadas tanto para pacientes com DGH sem reposição quanto para aqueles em reposição de $\mathrm{GH}$. Tem sido descrito que, em pacientes com DGH em reposição, as doses necessárias de reposição de levotiroxina estão aumentadas em relação àqueles sem reposição (70). Se esse aumento da reposição não for necessário, na verdade, esses pacientes podem estar tirotóxicos.

A otimização da reposição de levotiroxina é essencial, uma vez que mesmo a sub ou a super-reposição leve pode aumentar o risco de desenvolvimento de complicações. Isso é especialmente importante em pacientes com hipopituitarismo, visto que já foi demonstrado que esses pacientes apresentam maior mortalidade, especialmente cardiovascular (71). A reposição excessiva de levotiroxina pode contribuir com a osteoporose prematura, que está associada à deficiência de GH e gonadotrofinas (72-76), ao passo que a sub-reposição leve de levotiroxina pode aumentar o risco cardiovascular associado à deficiência de GH (77). Em resumo, embora a recomendação quanto à reposição de levotiroxina enfatize a necessidade de se evitar o hipotireoidismo deve ser notado que a super-reposição também deve ser evitada. Considerando que a avaliação da adequação da reposição da levotiroxina é de importância fundamental nos pacientes com hipopituitarismo, especialmente durante a infầncia, por causa de conseqüências deletérias tanto da sub quanto da super-reposição em relação ao crescimento e ao desenvolvimento normais, a recomendação quanto à reposição de levotiroxina 
deve ser avaliada adequadamente com base em marcadores de ação dos HT.

\section{AVALIAÇÃO DA REPOSIÇÃO TIREOIDIANA USANDO MARCADORES DE AÇÃO}

A adequação da reposição com levotiroxina deve ser avaliada idealmente por meio da mensuração de marcadores de ação do HT. $\mathrm{O} \mathrm{T}_{3}$, que é a forma bioativa do HT, age ligando-se a um receptor de HT específico nuclear (TR). O TR se liga ao DNA geralmente como um heterodímero com o receptor $\mathrm{X}$ retinóide (RXR) em seqüências específicas (elementos de resposta ao HT, TRE), os quais são ditados pelas preferências do sítio de ligação do DNA do complexo RXR-TR (ou TR-TR) (78). Dependendo do tecido examinado, essa ação evoca uma resposta que pode ser medida clínica (como a freqüência cardíaca) ou laboratorialmente.

No hipotireoidismo primário, o eixo hipotálamohipófise está intacto e os níveis séricos de TSH refletem precisamente a ação do HT na hipófise. $\mathrm{O}_{4}$ é convertido localmente em $\mathrm{T}_{3}$, o qual age no receptor de HT dentro das células hipofisárias e diminui a síntese e a liberação do TSH. Entretanto, uma vez que o defeito primário no HC está na unidade hipotálamo-hipófise, o TSH sérico não é marcador útil de ação do HT. Ao diagnóstico, os pacientes com hipopituitarismo podem se apresentar com níveis séricos de TSH baixos, normais ou discretamente aumentados. Os níveis aumentados de TSH (79) estão associados com a redução de sua bioatividade, provavelmente induzidos pelos níveis baixos de $\mathrm{T}_{4}(80)$. Após a reposição com levotiroxina, a maioria dos pacientes apresenta níveis suprimidos de TSH (18), levando à sugestão de que níveis séricos normais de TSH possam refletir reposição insuficiente. Embora essa sugestão possa estar correta para muitos pacientes, ela claramente não diferencia os pacientes com diferentes níveis de TSH pré-tratamento e também não pode distinguir aqueles pacientes repostos adequadamente daqueles com reposição excessiva. Os níveis séricos de $\mathrm{T}_{3}$ se mantêm dentro da faixa de referência em cerca de $25 \%$ dos pacientes com $\mathrm{T}_{4}$ livre baixo (81). Assim, o exame mais útil é o $\mathrm{T}_{4}$ livre, porém ele não é um marcador de ação tecidual do HT.

Muitos marcadores clínicos e séricos de ação do HT foram usados na prática clínica e testados em ensaios clínicos. Sinais e sintomas clássicos de hipotireoidismo são inespecíficos, principalmente em pacientes com deficiências hormonais concomitantes. Marcadores bioquímicos de ação periférica do HT, como colesterol, SHBG, enzima conversora de angiotensina, osteocalcina, telopeptídeo carboxi-terminal do colágeno tipo I e proteína óssea do ácido gama-carboxiglutâmico, se mostraram insuficientemente sensíveis e/ou específicos no diagnóstico e no manejo do HC (81).

Uma vez que o $\mathrm{T}_{3}$ modula a expressão genética de virtualmente todos os tecidos em vertebrados e o HT afeta a atividade de quase todos os tecidos em humanos, vários outros marcadores foram propostos. Entre os tecidos mais sensíveis, dois foram particularmente estudados e se mostraram sensíveis a mudanças no estado tirotrófico, o sistema cardiovascular e a termogênese.

\section{Sistema cardiovascular}

O HT tem várias ações no coração e no sistema vascular. Há muito tempo é reconhecido que o excesso ou a deficiência graves de HT induzem anormalidades, tanto na estrutura quanto na função cardíaca. Muitas das manifestações clínicas do hipertireoidismo são decorrentes da habilidade do HT de alterar a hemodinâmica cardiovascular (82). Os efeitos hemodinâmicos do hipotireoidismo são opostos aos do hipertireoidismo, embora sua manifestação clínica possa ser menos evidente.

Além dos efeitos bem conhecidos do HT no aumento do consumo periférico de oxigênio e de substratos, o que causa aumento secundário da contratilidade cardíaca, o HT também aumenta a contratilidade diretamente. O HT regula a expressão de vários genes que codificam proteínas cardíacas, incluindo a SERCa (adenosina trifosfatase cálcio do retículo sarcoplasmático), a cadeia pesada $\alpha$ da miosina, os receptores $\beta_{1}$ adrenérgi$\cos$ (positivamente), a cadeia pesada $\beta$ da miosina e a fosfolamban (negativamente) $(78,82,83)$.

O estado tireoidiano está ligado intimamente a mudanças na freqüência cardíaca, no débito cardíaco e na resistência vascular sistêmica $(78,82)$. Com o progresso nos estudos não-invasivos da função miocárdica em humanos, foram demonstradas alterações nos intervalos sistólicos em pacientes com hipo e hipertireoidismo, e a reversão dessas alterações após a normalização dos níveis de HT séricos (84-87). Especialmente interessante, os intervalos dos tempos sistólicos se mostraram acurados em diferenciar pacientes hipo, eu e hipertireóideos (85). 
Entre os tempos do intervalo sistólico, os dois mais usados são o período pré-ejetivo (tanto seu valor absoluto quanto o dividido pelo tempo de ejeção) e o tempo de contração isovolumétrica. Esse último é definido como o período entre o fechamento da valva mitral e a abertura da valva aórtica (88). O período pré-ejetivo também inclui o retardo eletromecânico, e foi usado mais freqüentemente quando os métodos empregados para medir os intervalos sistólicos incluíam a fonocardiografia $(85-87,89)$.

Usando o tempo de contração isovolumétrica como um marcador da ação do HT em pacientes com HC, nosso grupo observou prevalência alta de pacientes com HC e TCIV indicativo de deficiência de hormônio tereoidiano, apesar de níveis de $\mathrm{T}_{4}$ livre normais, sugerindo hipotireoidismo subclínico (90). A maioria desses pacientes com TCIV aumentado apresentou TCIV normal após ajuste da dose de levotiroxina. Vale ressaltar que a maioria desses pacientes era de adultos com deficiência de $\mathrm{GH}$ sem reposição de $\mathrm{GH}$ (88).

\section{Termogênese}

É muito conhecido, na prática clínica, que os pacientes com excesso ou deficiência de HT se queixem de intolerância ao calor ou ao frio, respectivamente. Esses sintomas são decorrentes da modulação da termogênese pelo HT (91). O HT também induz perda de peso apesar de ingesta calórica aumentada.

O HT afeta a termogênese por meio de vários mecanismos $(91,92)$. Estimula o metabolismo ao acelerar várias vias metabólicas sintéticas e catabólicas, as quais por sua vez aumentam a demanda por outros processos que requerem energia, como o transporte de metabólitos e íons por meio das membranas. O HT também é necessário para a termogênese facultativa, amplificando o efeito do sistema nervoso simpático em alvos termogenéticos, como o tecido adiposo marrom.

Em razão da inconveniência de se medir a produção de calor diretamente por meio de calorimetria direta, foram desenvolvidos métodos para a medição do gasto energético de repouso, expressão próxima da taxa metabólica basal. O gasto energético de repouso é medido em jejum, em repouso e em uma situação sem estresse por meio de calorimetria indireta. A quantidade de gases exalados e a percentagem de $\mathrm{O}_{2}$ e $\mathrm{CO}_{2}$ são transformados em kcal pela clássica equação de Weir (93).

Em pacientes com hipotireoidismo grave, os valores do gasto energético de repouso podem chegar a $-40 \%$ da faixa da normalidade, e em pacientes tirotóxi- cos os valores podem chegar a $+50 \%$ (94). Antes da disponibilidade dos ensaios para HT, a taxa metabólica basal era um dos testes mais importantes para a avaliação da função tireoidiana (95). A calorimetria indireta é um método simples, não-invasivo e bem tolerado pelos pacientes, além de ser de baixo custo (95).

Em um estudo que avaliou nove pacientes com hipotireoidismo e os estudou em três dosagens discretamente diferentes, variando entre o hiper e o hipotireoidismo subclínico (ou seja, com níveis de $\mathrm{T}_{4}$ livre normais), o gasto energético de repouso foi altamente sensível a essas pequenas mudanças, apresentando alta correlação com o TSH sérico basal, assim como com as mudanças no TSH séricos induzidas pelo ajuste da reposição com levotiroxina (96).

\section{Marcadores cardiovasculares e termogenéticos na avaliação de pacientes com deficiência combinada de GH e TSH}

Nosso grupo avaliou o impacto da reposição com GH no metabolismo do HT e as implicações desse efeito no manejo de pacientes com HC (32). Foram medidos os níveis séricos de $\mathrm{T}_{4}$ livre e $\mathrm{T}_{3}$ total, o tempo de contração isovolumétrica e o gasto energético de repouso em 32 pacientes e 23 controles. Desses pacientes 22 apresentavam $\mathrm{HC}$ em reposição com levotiroxina. Os pacientes com DGH em reposição com GH apresentaram níveis maiores de $\mathrm{T}_{3}$ total e marcadores de ação tireoidiana sugestivos de maior ação do HT quando comparados aos pacientes DGH sem reposição. Além disso, o $\mathrm{T}_{3}$ total, o TCIV e o GER no grupo com DGH reposto eram semelhantes aos controles. Quando foi testado o impacto do aumento da reposição de levotiroxina com o objetivo de manter níveis séricos de $\mathrm{T}_{4}$ livre na faixa superior da normalidade, tanto no grupo reposto com GH quanto no não reposto, os pacientes em reposição de GH tiveram maior risco de apresentar marcadores de ação tireoidiana compatíveis com tirotoxicose que os sem reposição (4 de 11 versus nenhum de sete pacientes). Níveis séricos de $T_{3}$ total foram associados a maior risco de reposição excessiva nesse grupo, conforme previamente sugerido por outros trabalhos $(33,81)$. Quando foi realizada a mesma avaliação em sete pacientes, tanto sem reposição de GH quanto após reposição, as mesmas mudanças ocorreram, ou seja, a reposição com GH diminuiu o $\mathrm{T}_{4}$ livre e o TCIV, e aumentou o $\mathrm{T}_{3}$ total e o GER, sugerindo que o GH aumenta o efeito biológico da tiroxina. Esses dados indicam que pacientes DGH com e sem reposição de GH devem ter 
alvos diferentes para a reposição de levotiroxina. Dessa maneira, sugerimos que pacientes sem reposição de DGH devam ser tratados conforme a recomendação atual, ou seja, mantendo o nível de $\mathrm{T}_{4}$ livre sérico na metade superior do intervalo de referência, uma vez que o DGH é um estado de hipoconversão de $\mathrm{T}_{4} \mathrm{em}$ $\mathrm{T}_{3}$. Por outro lado, pacientes em reposição de $\mathrm{GH}$ não apresentam nenhuma razão biológica para serem tratados para esse mesmo nível de $\mathrm{T}_{4}$ livre, e parece prudente ter como objetivo a média do intervalo de referência, de maneira semelhante a indivíduos normais. $\mathrm{Na}$ verdade, níveis na faixa superior da normalidade de $\mathrm{T}_{4}$ livre podem aumentar o risco de super-reposição, o que pode ser tão deletério quanto a sub-reposição.

\section{GH E EIXO CORTICOTRÓFICO}

É interessante notar que algumas características clínicas e laboratoriais associadas à deficiência de hormônio de crescimento são comuns em pacientes com hipercortisolismo, em especial o aumento de tecido adiposo visceral e da resistência insulínica. Dessa forma, a inter-relação entre o eixo somatotrófico e o eixo corticotrófico despertou especial interesse, sendo levantada a hipótese de que um dos mecanismos que poderia explicar o efeito benéfico da reposição de $\mathrm{GH}$ em pacientes hiposomatotróficos poderia ser a modulação do eixo corticotrófico induzida pelo GH/IGF-1.

Nenhum efeito do GH na secreção de cortisol foi observado em humanos (97). A administração de GH em doses altas pode reduzir a concentração da globulina ligadora de cortisol (CBG) $(98,99)$, porém nenhum efeito foi encontrado após doses menores (100). Além disso, nenhum efeito foi encontrado nas redutases do anel A ( $5 \alpha$-redutase e $5 \beta$-redutase) $(100,101)$.

A interconversão do cortisol (hormonalmente ativo) em cortisona (hormonalmente inativa) é mediada por duas isoenzimas da $11 \beta$-hidroxiesteróide desidrogenase (11 $\beta$-HSD), a tipo 1 (ativando a cortisona) e a tipo 2 (inativando o cortisol). A $11 \beta$-HSD tipo 1 está presente principalmente no fígado, no tecido adiposo e nas gônadas, enquanto a tipo 2 encontra-se principalmente nos túbulos renais e no cólon.

Vários estudos demonstraram que a administração de GH acelera o metabolismo do cortisol $(98,101)$. Em pacientes com deficiência combinada de GH e ACTH, a reposição de $\mathrm{GH}$ induz a diminuição significativa da expressão de RNAm de $11 \beta$-HSDl e aumento da expres- são de $11 \beta-H S D 2$ em tecido adiposo subcutâneo. O efeito máximo do $\mathrm{GH}$ no metabolismo periférico dos glicocorticóides em pacientes com hipopituitarismo ocorre com doses baixas de $\mathrm{GH}$ e é independente de mudanças nos níveis séricos de insulina ou na massa adiposa (102). Esse aumento da atividade da $11 \beta$-HSDl resulta aumento da produção de cortisol localmente, tanto no fígado quanto na gordura visceral, o que pode explicar o desenvolvimento de adiposidade visceral e a resistência insulínica, típicos do DGH.

Um estudo avaliou os efeitos da reposição de GH em pacientes com DGH e aparente reserva adrenal normal quanto aos efeitos dessa reposição no estado corticotrófico (103). Nesse estudo, 12 pacientes com DGH foram avaliados antes e depois da reposição de GH com doses-padrão. Desses 12 , nove apresentaram evidências bioquímicas de insuficiência corticotrófica, apesar de se manterem assintomáticos. A maioria dos pacientes avaliados apresentava múltiplas deficiências hipofisárias. Outro estudo do mesmo grupo avaliando pacientes com DGH isolado não mostrou alteração do eixo corticotrófico (104). Em conjunto, esses dados sugerem que em pacientes com reserva normal de ACTH, o que ocorre em casos de DGH isolado, a redução da disponibilidade sistêmica de glicocorticóide é compensada pelo aumento da secreção de ACTH. Tal mecanismo não ocorre em pacientes com múltiplas deficiências, provavelmente portadores de insuficiência adrenocorticotrófica parcial. Assim, é essencial que os pacientes sejam avaliados após o início da reposição com $\mathrm{GH}$, em especial aqueles com múltiplas deficiências, pelo risco de precipitação de insuficiência adrenal.

Dessa forma, o aumento da obesidade visceral e da resistência insulínica associado ao DGH podem ser parcialmente explicados pelo aumento da atividade da $11 \beta$-HSD 1, o que leva à maior disponibilidade do cortisol no tecido adiposo e nos hepatócitos. A normalização da conversão de cortisol em cortisona seria responsável em parte pelos efeitos benéficos da reposição de GH nesses pacientes.

O efeito do GH estimulando a inativação do cortisol pode também influenciar os efeitos da reposição glicocorticóide. Pacientes com deficiência combinada de GH e ACTH em reposição com hidrocortisona e pacientes em reposição com acetato de hidrocortisona foram avaliados tanto no estado pré quanto no pós-reposição com GH (100). Os resultados sugeriram que os pacientes recebendo acetato de cortisona podem ter exposição tecidual mais fisiológica de glicocorticóide 
enquanto não estão recebendo reposição de $\mathrm{GH}$, mas estão mais vulneráveis à deficiência glicocorticóide durante o tratamento com $\mathrm{GH}$.

\section{CONCLUSÕES}

O estado somatotrófico é determinante importante do estado tirotrófico. A reposição de GH aumenta a conversão de $\mathrm{T}_{4}$ em $\mathrm{T}_{3}$. Assim, os pacientes com $\mathrm{HC}$ devem ser tratados de acordo com o seu estado somatotrófico. Os pacientes com DGH não-repostos devem ser tratados conforme as recomendações tradicionais, ou seja, mantendo níveis séricos de $\mathrm{T}_{4}$ livre na metade superior do intervalo de referência. No entanto, os pacientes em reposição de GH devem ser mantidos com seu $\mathrm{T}_{4}$ livre próximo da média do intervalo de referência, a fim de se evitar tirotoxicose. Os níveis séricos de $T_{3}$ devem ser medidos durante o seguimento para ajudar e detectar reposição excessiva. A avaliação de marcadores de ação tecidual dos HT, como o TCIV e o GER, pode ajudar a detectar o nível ótimo de $\mathrm{T}_{4}$ livre em determinado paciente.

É interessante notar que em contraste com o efeito do GH no eixo tirotrófico, aumentando a forma ativa do HT, no eixo corticotrófico a reposição de GH tem efeito contrário. É importante reavaliar o eixo corticotrófico de pacientes com deficiência de GH após o início da reposição somatotrófica. O risco de insuficiência adrenal é maior em pacientes com múltiplas deficiências hipofisárias, ao passo que em pacientes com DGH isolado esse risco é bem menor.

\section{REFERÊNCIAS}

1. Beck-Peccoz P, Amr S, Menezes-Ferreira MM, Faglia G, Weintraub BD. Decreased receptor binding of biologically inactive thyrotropin in central hypothyroidism. Effect of treatment with thyrotropin-releasing hormone. N Engl J Med. 1985;312(17): 1085-90.

2. Oliveira JH, Persani L, Beck-Peccoz P, Abucham J. Investigating the paradox of hypothyroidism and increased serum thyrotropin (TSH) levels in Sheehan's syndrome: characterization of TSH carbohydrate content and bioactivity. J Clin Endocrinol Metab. 2001;86(4):1694-9.

3. Faglia G, Bitensky L, Pinchera A, Ferrari C, Paracchi A, BeckPeccoz $P$, et al. Thyrotropin secretion in patients with central hypothyroidism: evidence for reduced biological activity of immunoreactive thyrotropin. J Clin Endocrinol Metab. 1979;48(6):989-98.

4. Maison P, Chanson P. Cardiac effects of growth hormone in adults with growth hormone deficiency: a meta-analysis. Circulation. 2003;108(21):2648-52.
5. Benvenga S, Campenni A, Ruggeri RM, Trimarchi F. Clinical review 113: hypopituitarism secondary to head trauma. J Clin Endocrinol Metab. 2000;85(4):1353-61.

6. Bondanelli M, Ambrosio MR, Onofri A, Bergonzoni A, Lavezzi $\mathrm{S}$, Zatelli MC, et al. Predictive value of circulating insulin-like growth factor I levels in ischemic stroke outcome. J Clin Endocrinol Metab. 2006;91(10):3928-34.

7. Schneider HJ, Rovere S, Corneli G, Croce CG, Gasco V, Ruda $\mathrm{R}$, et al. Endocrine dysfunction in patients operated on for non-pituitary intracranial tumors. Eur J Endocrinol. 2006; 155(4):559-66.

8. Agha A, Sherlock M, Brennan S, O'Connor SA, O'Sullivan E, Rogers B, et al. Hypothalamic-pituitary dysfunction after irradiation of nonpituitary brain tumors in adults. J Clin Endocrinol Metab. 2005;90(12):6355-60.

9. Partsch CJ, Riepe FG, Krone N, Sippell WG, Pohlenz J. Initially elevated TSH and congenital central hypothyroidism due to a homozygous mutation of the TSH beta subunit gene: case report and review of the literature. Exp Clin Endocrinol Diabetes. 2006; 114(5):227-34.

10. Hayashizaki $Y$, Hiraoka $Y$, Endo $Y$, Miyai $K$, Matsubara $K$. Thyroid-stimulating hormone (TSH) deficiency caused by a single base substitution in the CAGYC region of the beta-subunit. EMBO J. 1989;8(8):2291-6.

11. Dacou-Voutetakis C, Feltquate DM, Drakopoulou M, Kourides IA, Dracopoli NC. Familial hypothyroidism caused by a nonsense mutation in the thyroid-stimulating hormone beta-subunit gene. Am J Hum Genet. 1990;46(5):988-93.

12. Medeiros-Neto G, Herodotou DT, Rajan S, Kommareddi S, de Lacerda $L$, Sandrini $R$, et al. A circulating, biologically inactive thyrotropin caused by a mutation in the beta subunit gene. $J$ Clin Invest. 1996;97(5):1250-6.

13. Doeker BM, Pfaffle RW, Pohlenz J, Andler W. Congenital central hypothyroidism due to a homozygous mutation in the thyrotropin beta-subunit gene follows an autosomal recessive inheritance. J Clin Endocrinol Metab. 1998;83(5):1762-5.

14. Collu R, Tang J, Castagne J, Lagace G, Masson N, Huot C, et al. A novel mechanism for isolated central hypothyroidism: inactivating mutations in the thyrotropin-releasing hormone receptor gene. J Clin Endocrinol Metab. 1997;82(5):1561-5.

15. Vieira TC, Boldarine VT, Abucham J. Molecular analysis of PROP1, PIT1, HESX1, LHX3, and LHX4 shows high frequency of PROP1 mutations in patients with familial forms of combined pituitary hormone deficiency. Arq Bras Endocrinol Metabol. 2007;51(7):1097-103.

16. Wu W, Cogan JD, Pfaffle RW, Dasen JS, Frisch H, O'Connell $\mathrm{SM}$, et al. Mutations in PROP1 cause familial combined pituitary hormone deficiency. Nat Genet. 1998;18(2):147-9.

17. Mehta A, Hindmarsh PC, Stanhope RG, Brain CE, Preece MA, Dattani MT. Is the thyrotropin-releasing hormone test necessary in the diagnosis of central hypothyroidism in children. $J$ Clin Endocrinol Metab. 2003;88(12):5696-703.

18. Shimon I, Cohen O, Lubetsky A, Olchovsky D. Thyrotropin suppression by thyroid hormone replacement is correlated with thyroxine level normalization in central hypothyroidism. Thyroid. 2002;12(9):823-7.

19. Andersen S, Pedersen KM, Bruun NH, Laurberg P. Narrow individual variations in serum $\mathrm{T}(4)$ and $\mathrm{T}(3)$ in normal subjects: a clue to the understanding of subclinical thyroid disease. $J$ Clin Endocrinol Metab. 2002;87(3):1068-72.

20. Darras VM, Berghman LR, Vanderpooten A, Kuhn ER. Growth hormone acutely decreases type III iodothyronine deiodinase in chicken liver. FEBS Lett. 1992;310(1):5-8. 
21. Carrozza V, Csako G, Yanovski JA, Skarulis MC, Nieman L, Wesley $R$, et al. Levothyroxine replacement therapy in central hypothyroidism: a practice report. Pharmacotherapy. 1999;19(3):349-55.

22. Schneider HJ, Aimaretti G, Kreitschmann-Andermahr I, Stalla GK, Ghigo E. Hypopituitarism. Lancet. 2007;369(9571): 1461-70.

23. Lippe BM, Van Herle AJ, LaFranchi SH, Uller RP, Lavin N, Kaplan SA. Reversible hypothyroidism in growth hormone-deficient children treated with human growth hormone. J Clin Endocrinol Metab. 1975;40(4):612-8.

24. Root AW, Bongiovanni AM, Eberlein WR. Inhibition of thyroidal radioiodine uptake by human growth hormone. J Pediatr. 1970;76(3):422-9.

25. Root AW, Snyder PJ, Rezvani I, DiGeorge AM, Utiger RD. Inhibition of thyrotropin releasing hormone-mediated secretion of thyrotropin by human growth hormone. J Clin Endocrinol Metab. 1973;36(1):103-7.

26. Bray GA, Raben MS, Londono J, Gallagher TF Jr. Effects of triiodothyronine, growth hormone and anabolic steroids on nitrogen excretion and oxygen consumption of obese patients. J Clin Endocrinol Metab. 1971;33(2):293-300.

27. Moller J, Jorgensen JO, Moller N, Christiansen JS, Weeke J. Effects of growth hormone administration on fuel oxidation and thyroid function in normal man. Metabolism. 1992; 41(7):728-31.

28. Sato T, Suzukui Y, Taketani T, Ishiguro K, Masuyama T. Enhanced peripheral conversion of thyroxine to triiodothyronine during hGH therapy in $\mathrm{GH}$ deficient children. J Clin Endocrinol Metab. 1977;45(2):324-9.

29. Rezvani I, DiGeorge AM, Dowshen SA, Bourdony CJ. Action of human growth hormone ( $\mathrm{hGH}$ ) on extrathyroidal conversion of thyroxine (T4) to triiodothyronine (T3) in children with hypopituitarism. Pediatr Res. 1981;15(1):6-9.

30. Grunfeld C, Sherman BM, Cavalieri RR. The acute effects of human growth hormone administration on thyroid function in normal men. J Clin Endocrinol Metab. 1988;67(5):1111-4.

31. Jorgensen JO, Moller J, Laursen T, Orskov H, Christiansen JS, Weeke J. Growth hormone administration stimulates energy expenditure and extrathyroidal conversion of thyroxine to triiodothyronine in a dose-dependent manner and suppresses circadian thyrotrophin levels: studies in $\mathrm{GH}$-deficient adults. Clin Endocrinol (Oxf). 1994;41(5):609-14.

32. Martins MR, Doin FC, Komatsu WR, Barros-Neto TL, Moises VA, Abucham J. Growth hormone replacement improves thyroxine biological effects: implications for management of central hypothyroidism. J Clin Endocrinol Metab. 2007; 92(11):4144-53.

33. Portes ES, Oliveira JH, MacCagnan P, Abucham J. Changes in serum thyroid hormones levels and their mechanisms during long-term growth hormone $(\mathrm{GH})$ replacement therapy in $\mathrm{GH}$ deficient children. Clin Endocrinol (Oxf). 2000;53(2):183-9.

34. Pirazzoli P, Cacciari E, Mandini M, Sganga T, Capelli M, Cicognani $A$, et al. Growth and thyroid function in children treated with growth hormone. J Pediatr. 1992;121(2):210-3.

35. Jorgensen JO, Pedersen SA, Laurberg P, Weeke J, Skakkebaek NE, Christiansen JS. Effects of growth hormone therapy on thyroid function of growth hormone-deficient adults with and without concomitant thyroxine-substituted central hypothyroidism. J Clin Endocrinol Metab. 1989; 69(6):1127-32.

36. Porter BA, Refetoff $S$, Rosenfeld RL, De Groat LJ, Lang US, Stark O. Abnormal thyroxine metabolism in hyposomatotro- phic dwarfism and inhibition of responsiveness to TRH during GH therapy. Pediatrics. 1973;51(4):668-74.

37. Rubio GR, Mellinger RC, Zafar MS, Wolf CB. Evaluation of thyroid function during growth hormone therapy. Metabolism. 1976;25(1):15-21

38. Cacciari E, Cicognani A, Pirazzoli P, Bernardi F, Zappulla F, Salardi $\mathrm{S}$, et al. Effect of long-term GH administration on pituitary-thyroid function in idiopathic hypopituitarism. Acta Paediatr Scand. 1979;68(3):405-9.

39. Municchi G, Malozowski S, Nisula BC, Cristiano A, Rose SR. Nocturnal thyrotropin surge in growth hormone-deficient children. J Pediatr. 1992;121(2):214-20.

40. Rose SR, Leong GM, Yanovski JA, Blum D, Heavner G, Barnes $\mathrm{KM}$, et al. Thyroid function in non-growth hormone-deficient short children during a placebo-controlled double blind trial of recombinant growth hormone therapy. J Clin Endocrinol Metab. 1995;80(1):320-4.

41. Amato G, Izzo G, Salzano I, Bellastella A. Recombinant human growth hormone treatment at low doses does not significantly change thyroid function in growth hormone deficient adults. J Endocrinol Invest. 1996;19(8):563-6.

42. Hussain MA, Schmitz O, Jorgensen JO, Christiansen JS, Weeke J, Schmid C, et al. Insulin-like growth factor I alters peripheral thyroid hormone metabolism in humans: comparison with growth hormone. Eur J Endocrinol. 1996;134(5):563-7.

43. Wyatt DT, Gesundheit N, Sherman B. Changes in thyroid hormone levels during growth hormone therapy in initially euthyroid patients: lack of need for thyroxine supplementation. J Clin Endocrinol Metab. 1998;83(10):3493-7.

44. Giavoli C, Porretti S, Ferrante E, Cappiello V, Ronchi CL, Travaglini $\mathrm{P}$, et al. Recombinant $\mathrm{hGH}$ replacement therapy and the hypothalamus-pituitary-thyroid axis in children with GH deficiency: when should we be concerned about the occurrence of central hypothyroidism? Clin Endocrinol (Oxf). 2003;59(6): 806-10.

45. Kalina-Faska B, Kalina M, Koehler B. Effects of recombinant growth hormone therapy on thyroid hormone concentrations. Int J Clin Pharmacol Ther. 2004;42(1):30-4.

46. Seminara S, Stagi S, Candura L, Scrivano M, Lenzi L, Nanni L, et al. Changes of thyroid function during long-term hGH therapy in GHD children. A possible relationship with catch-up growth? Horm Metab Res. 2005;37(12):751-6.

47. Binnerts A, Swart GR, Wilson JH, Hoogerbrugge N, Pols HA, Birkenhager JC, et al. The effect of growth hormone administration in growth hormone deficient adults on bone, protein, carbohydrate and lipid homeostasis, as well as on body composition. Clin Endocrinol (Oxf). 1992;37(1):79-87.

48. Bengtsson BA, Eden S, Lonn L, Kvist $H$, Stokland A, Lindstedt $\mathrm{G}$, et al. Treatment of adults with growth hormone $(\mathrm{GH})$ deficiency with recombinant human GH. J Clin Endocrinol Metab. 1993;76(2):309-17.

49. Jorgensen JO, Pedersen SB, Borglum J, Moller N, Schmitz O, Christiansen JS, et al. Fuel metabolism, energy expenditure, and thyroid function in growth hormone-treated obese women: a double-blind placebo-controlled study. Metabolism. 1994;43(7):872-7.

50. Porretti S, Giavoli C, Ronchi C, Lombardi G, Zaccaria M, Valle $D$, et al. Recombinant human GH replacement therapy and thyroid function in a large group of adult GH-deficient patients: when does L-T(4) therapy become mandatory? J Clin Endocrinol Metab. 2002;87(5):2042-5.

51. Giavoli C, Cappiello V, Porretti S, Ronchi CL, Orsi E, Beck-Peccoz P, et al. Growth hormone therapy in $\mathrm{GH}$-deficient adults: 
continuous vs alternate-days treatment. Horm Metab Res. 2003;35(9):557-61.

52. Bianco AC, Salvatore D, Gereben B, Berry MJ, Larsen PR. Biochemistry, cellular and molecular biology, and physiological roles of the iodothyronine selenodeiodinases. Endocr Rev. 2002;23(1):38-89.

53. Kuiper GG, Kester MH, Peeters RP, Visser TJ. Biochemical mechanisms of thyroid hormone deiodination. Thyroid. 2005;15(8):787-98.

54. Bianco AC, Kim BW. Deiodinases: implications of the local control of thyroid hormone action. J Clin Invest. 2006;116(10): 2571-9.

55. Maia AL, Kim BW, Huang SA, Harney JW, Larsen PR. Type 2 iodothyronine deiodinase is the major source of plasma T3 in euthyroid humans. J Clin Invest. 2005;115(9):2524-33.

56. Maia AL. Type 1 iodothyronine deiodinase is the major source of circulating T3 in hyperthyroidism: implications for therapy. Nat Clin Pract Endocrinol Metab. 2007;3(11):740-1.

57. de Luze A, Leloup J. Fish growth hormone enhances peripheral conversion of thyroxine to triiodothyronine in the eel (Anguilla anguilla L.). Gen Comp Endocrinol. 1984;56(2):308-12.

58. MacLatchy DL, Eales JG. Growth hormone stimulates hepatic thyroxine $5^{\prime}$-monodeiodinase activity and 3,5,3'-triiodothyronine levels in rainbow trout (Salmo gairdneri). Gen Comp Endocrinol. 1990;78(2):164-72.

59. Kuhn ER, Verheyen G, Chiasson RB, Huts C, Huybrechts L, Van den Steen P, et al. Growth hormone stimulates the peripheral conversion of thyroxine into triiodothyronine by increasing the liver $5^{\prime}$-monodeiodinase activity in the fasted and normal fed chicken. Horm Metab Res. 1987;19(7):304-8.

60. Kuhn ER, Van Osselaer P, Siau O, Decuypere E, Moreels A. Thyroid function in newborn lambs: influence of prolactin and growth hormone. J Endocrinol. 1986;109(2):215-9.

61. Rumsey TS, Kahl S, Norton SA, Eisemann J, Elsasser TH, Hammond AC, et al. Evidence that bovine growth hormone treatment increases the rate of extrathyroidal $5^{\prime}$-monodeiodinase activity in cattle. Domest Anim Endocrinol. 1990;7(2): 125-34.

62. Iqbal A, Cheema AM, Kuhn ER. Growth hormone induced stimulation of the T4 to T3 conversion in fed and fasting dwarf goats. Horm Metab Res. 1990;22(11):566-8.

63. Berghman L, Darras VM, Huybrechts LM, Decuypere E, Vandesande F, Kuhn ER. Evidence for chicken GH as the only hypophyseal factor responsible for the stimulation of hepatic 5 '-monodeiodination activity in the chick embryo. Reprod Nutr Dev. 1989;29(2):197-202

64. Van der Geyten S, Buys N, Sanders JP, Decuypere E, Visser TJ, Kuhn ER, et al. Acute pretranslational regulation of type III iodothyronine deiodinase by growth hormone and dexamethasone in chicken embryos. Mol Cell Endocrinol. 1999;147(1-2): 49-56.

65. Kuhn ER, Shimada K, Ohkubo T, Vleurick LM, Berghman LR, Darras VM. Influence of recombinant chicken prolactin on thyroid hormone metabolism in the chick embryo. Gen Comp Endocrinol. 1996;103(3):349-58.

66. Schwander JC, Hauri C, Zapf J, Froesch ER. Synthesis and secretion of insulin-like growth factor and its binding protein by the perfused rat liver: dependence on growth hormone status. Endocrinology. 1983;113(1):297-305.

67. Ballesteros M, Leung KC, Ross RJ, lismaa TP, Ho KK. Distribution and abundance of messenger ribonucleic acid for growth hormone receptor isoforms in human tissues. J Clin Endocrinol Metab. 2000;85(8):2865-71.
68. Caro JF, Poulos J, Ittoop O, Pories WJ, Flickinger EG, Sinha MK. Insulin-like growth factor I binding in hepatocytes from human liver, human hepatoma, and normal, regenerating, and fetal rat liver. J Clin Invest. 1988;81(4):976-81.

69. Klinger B, lonesco A, Anin S, Laron Z. Effect of insulin-like growth factor I on the thyroid axis in patients with Laron-type dwarfism and healthy subjects. Acta Endocrinol (Copenh). 1992;127(6):515-9.

70. Alexopoulou O, Beguin C, De Nayer P, Maiter D. Clinical and hormonal characteristics of central hypothyroidism at diagnosis and during follow-up in adult patients. Eur J Endocrinol. 2004;150(1):1-8.

71. Tomlinson JW, Holden N, Hills RK, Wheatley K, Clayton RN, Bates AS et al. Association between premature mortality and hypopituitarism. West Midlands Prospective Hypopituitary Study Group. Lancet. 2001;357(9254):425-31.

72. Toft AD. Thyroxine therapy. N Engl J Med. 1994;331(3):174-80.

73. Mandel SJ, Brent GA, Larsen PR. Levothyroxine therapy in patients with thyroid disease. Ann Intern Med. 1993;119(6): 492-502.

74. Campos-Pastor MM, Munoz-Torres M, Escobar-Jimenez F, Ruiz de Almodovar M, Jodar Gimeno E. Bone mass in females with different thyroid disorders: influence of menopausal status. Bone Miner. 1993;21(1):1-8.

75. Solomon BL, Wartofsky L, Burman KD. Prevalence of fractures in postmenopausal women with thyroid disease. Thyroid. 1993;3(1):17-23.

76. Mosekilde L, Eriksen EF, Charles P. Effects of thyroid hormones on bone and mineral metabolism. Endocrinol Metab Clin North Am. 1990;19(1):35-63.

77. Kahaly GJ. Cardiovascular and atherogenic aspects of subclinical hypothyroidism. Thyroid. 2000;10(8):665-79.

78. Kahaly GJ, Dillmann WH. Thyroid hormone action in the heart. Endocr Rev. 2005;26(5):704-28.

79. Abucham J, Castro V, Maccagnan P, Vieira JG. Increased thyrotrophin levels and loss of the nocturnal thyrotrophin surge in Sheehan's syndrome. Clin Endocrinol (Oxf). 1997;47(5): 515-22.

80. Oliveira JH, Barbosa ER, Kasamatsu T, Abucham J. Evidence for thyroid hormone as a positive regulator of serum thyrotropin bioactivity. J Clin Endocrinol Metab. 2007;92(8):3108-13.

81. Ferretti E, Persani L, Jaffrain-Rea ML, Giambona S, Tamburrano G, Beck-Peccoz P. Evaluation of the adequacy of levothyroxine replacement therapy in patients with central hypo-thyroidism. J Clin Endocrinol Metab. 1999;84(3):924-9.

82. Klein I, Ojamaa K. Thyroid hormone and the cardiovascular system. N Engl J Med. 2001;344(7):501-9.

83. Yen PM. Physiological and molecular basis of thyroid hormone action. Physiol Rev. 2001;81(3):1097-142.

84. Crowley WF Jr, Ridgway EC, Bough EW, Francis GS, Daniels $\mathrm{GH}$, Kourides IA, et al. Noninvasive evaluation of cardiac function in hypothyroidism. Response to gradual thyroxine replacement. N Engl J Med. 1977;296(1):1-6.

85. Hillis WS, Bremner WF, Lawrie TD, Thomson JA. Systolic time intervals in thyroid disease. Clin Endocrinol (Oxf). 1975;4(6): 617-24.

86. Amidi M, Leon DF, DeGroot WJ, Kroetz FW, Leonard JJ. Effect of the thyroid state on myocardial contractility and ventricular ejection rate in man. Circulation. 1968;38(2):229-39.

87. Young RT, Van Herle AJ, Rodbard D. Improved diagnosis and management of hyper- and hypothyroidism by timing the arterial sounds. J Clin Endocrinol Metab. 1976;42(2):330-40. 
88. Doin FL, Borges Mda R, Campos O, de Camargo Carvalho AC, de Paola AA, Paiva MG, et al. Effect of central hypothyroidism on Doppler-derived myocardial performance index. J Am Soc Echocardiogr. 2004;17(6):622-9.

89. Parisi AF, Hamilton BP, Thomas CN, Mazzaferri EL. The short cardiac pre-ejection period: an index to thyrotoxicosis. Circulation. 1974;49(5):900-4.

90. Borges MR, Doin FL, Moises VA, Abucham J. Isovolumic contraction time of the heart in the diagnosis and therapeutic management of central hypothyroidism. Annals of the 12th International Congress of Endocrinology. 2004;1191-6.

91. Silva JE. The multiple contributions of thyroid hormone to heat production. J Clin Invest. 2001;108(1):35-7.

92. Silva JE. The thermogenic effect of thyroid hormone and its clinical implications. Ann Intern Med. 2003;139(3):205-13.

93. Weir JB. New methods for calculating metabolic rate with special reference to protein metabolism. J Physiol. 1949;109(1-2):1-9.

94. Freake HC, Oppenheimer JH. Thermogenesis and thyroid function. Annu Rev Nutr. 1995;15:263-91.

95. Lim VS, Zavala DC, Flanigan MJ, Freeman RM. Basal oxygen uptake: a new technique for an old test. J Clin Endocrinol Metab. 1986;62(5):863-8.

96. al-Adsani $\mathrm{H}$, Hoffer LJ, Silva JE. Resting energy expenditure is sensitive to small dose changes in patients on chronic thyroid hormone replacement. J Clin Endocrinol Metab. 1997;82(4): 1118-25.

97. Gianotti L, Ramunni J, Lanfranco F, Maccagno B, Giordano R, Broglio F, et al. Recombinant human IGF-I does not modify the $\mathrm{ACTH}$ and cortisol responses to hCRH and hexarelin, a peptidyl GH secretagogue, in humans. J Endocrinol Invest. 2001;24(2):67-71.

98. Weaver JU, Thaventhiran L, Noonan K, Burrin JM, Taylor NF, Norman MR, et al. The effect of growth hormone replacement on cortisol metabolism and glucocorticoid sensitivity in hypopituitary adults. Clin Endocrinol (Oxf). 1994;41(5): 639-48.

99. Rodriguez-Arnao J, Perry L, Besser GM, Ross RJ. Growth hormone treatment in hypopituitary GH deficient adults reduces circulating cortisol levels during hydrocortisone replacement therapy. Clin Endocrinol (Oxf). 1996;45(1):33-7.

100. Swords FM, Carroll PV, Kisalu J, Wood PJ, Taylor NF, Monson JP. The effects of growth hormone deficiency and replacement on glucocorticoid exposure in hypopituitary patients on cortisone acetate and hydrocortisone replacement. Clin Endocrinol (Oxf). 2003;59(5):613-20.

101. Gelding SV, Taylor NF, Wood PJ, Noonan K, Weaver JU, Wood DF, et al. The effect of growth hormone replacement therapy on cortisol-cortisone interconversion in hypopituitary adults: evidence for growth hormone modulation of extrarenal 11 beta-hydroxysteroid dehydrogenase activity. Clin Endocrinol (Oxf). 1998;48(2):153-62.

102. Toogood AA, Taylor NF, Shalet SM, Monson JP. Modulation of cortisol metabolism by low-dose growth hormone replacement in elderly hypopituitary patients. J Clin Endocrinol Metab. 2000;85(4):1727-30.

103. Giavoli C, Libe R, Corbetta S, Ferrante E, Lania A, Arosio M, et al. Effect of recombinant human growth hormone (GH) replacement on the hypothalamic-pituitary-adrenal axis in adult GH-deficient patients. J Clin Endocrinol Metab. 2004; 89(11):5397-401.

104. Giavoli $C$, Bergamaschi S, Ferrante E, Ronchi CL, Lania AG, Rusconi R, et al. Effect of growth hormone deficiency and recombinant $\mathrm{hGH}(\mathrm{hrGH})$ replacement on the hypothalamicpituitary-adrenal axis in children with idiopathic isolated GH deficiency. Clin Endocrinol (Oxf). 2008;68(2):247-51.

\section{Endereço para correspondência:}

Julio Abucham

Disciplina de Endocrinologia EPM/Unifesp

Rua Pedro de Toledo, 910

04039-002 São Paulo SP

E-mail: julioabucham@uol.com.br 